OPEN ACCESS

Edited by:

Gabriele Berg,

Graz University of Technology, Austria

Reviewed by:

Yvonne Vallès,

New York University Abu Dhabi, UAE

Renee Maxine Petri,

Veterinärmedizinische Universität,

Austria

${ }^{*}$ Correspondence: Barbara Klug

barbara.klug@medunigraz.at

Specialty section:

This article was submitted to

Microbial Symbioses,

a section of the journal

Frontiers in Microbiology

Received: 30 April 2016 Accepted: 19 December 2016 Published: 18 January 2017

Citation:

Santigli E, Trajanoski S, Eberhard K and Klug B (2017) Sampling Modification Effects in the Subgingival Microbiome Profile of Healthy

Children. Front. Microbiol. 7:2142. doi: 10.3389/fmicb.2016.02142

\section{Sampling Modification Effects in the Subgingival Microbiome Profile of Healthy Children}

\author{
Elisabeth Santigli ${ }^{1}$, Slave Trajanoski ${ }^{2}$, Katharina Eberhard ${ }^{2}$ and Barbara Klug ${ }^{1 *}$ \\ ${ }^{1}$ Division of Oral Surgery and Orthodontics, Department of Dental Medicine and Oral Health, Medical University of Graz, \\ Graz, Austria, ${ }^{2}$ Center for Medical Research, Medical University of Graz, Graz, Austria
}

Background: Oral microbiota are considered major players in the development of periodontal diseases. Thorough knowledge of intact subgingival microbiomes is required to elucidate microbial shifts from health to disease.

Aims: This comparative study investigated the subgingival microbiome of healthy children, possible inter- and intra-individual effects of modified sampling, and basic comparability of subgingival microprints.

Methods: In five 10-year-old children, biofilm was collected from the upper first premolars and first molars using sterilized, UV-treated paper-points inserted into the subgingival sulcus at eight sites. After supragingival cleaning using an electric toothbrush and water, sampling was performed, firstly, excluding (Mode A) and, secondly, including (Mode B) cleansing with sterile cotton pellets. DNA was extracted from the pooled samples, and primers targeting 16S rRNA hypervariable regions V5 and V6 were used for 454-pyrosequencing. Wilcoxon signed rank test and $t$-test were applied to compare sampling modes. Principal coordinate analysis (PCOA) and average agglomerative hierarchical clustering were calculated with unweighted UniFrac distance matrices. Sample grouping was tested with permutational MANOVA (Adonis).

Results: Data filtering and quality control yielded 67,218 sequences with an average sequence length of 243bp (SD 6.52; range 231-255). Actinobacteria (2.8-24.6\%), Bacteroidetes (9.2-25.1\%), Proteobacteria (4.9-50.6\%), Firmicutes (16.5-57.4\%), and Fusobacteria (2.2-17.1\%) were the five major phyla found in all samples. Differences in microbial abundances between sampling modes were not evident. High sampling numbers are needed to achieve significance for rare bacterial phyla. Samples taken from one individual using different sampling modes were more similar to each other than to other individuals' samples. PCOA and hierarchical clustering showed a grouping of the paired samples. Permutational MANOVA did not reveal sample grouping by sampling modes $\left(p=0.914\right.$ by $\left.R^{2}=0.09\right)$.

Conclusion: A slight modification of sampling mode has minor effects corresponding to a natural variability in the microbiome profiles of healthy children. The inter-individual variability in subgingival microprints is greater than intra-individual differences. Statistical 
analyses of microbial populations should consider this baseline variability and move beyond mere quantification with input from visual analytics. Comparative results are difficult to summarize as methods for studying huge datasets are still evolving. Advanced approaches are needed for sample size calculations in clinical settings.

Keywords: oral microbiome, subgingival biofilm, healthy children, next generation sequencing (NGS), 454-pyrosequencing, paper point, subgingival sampling

\section{INTRODUCTION}

Oral bacterial biofilm research is an emerging field. During the last decades, the profiling of oral microbial communities has evolved from bacterial culture experiments to biofilm characterization by detailed classification using cultureindependent methods (Jenkinson, 2011; Diaz, 2012; Simón-Soro et al., 2013). High throughput next generation sequencing (NGS) like 454-pyrosequencing and metagenome analysis have replaced fingerprinting methods (Ahn et al., 2011; Griffen et al., 2011; Alcaraz et al., 2012; Li et al., 2012, 2013; Siqueira et al., 2012; Abusleme et al., 2013; Trajanoski et al., 2013; Chen et al., 2015; Park et al., 2015). Instead of identifying single bacteria, operational taxonomic units (OTUs) based on sequence similarities (of mostly 97\%) are assigned to identify groups of bacteria. This has led to a new research avenue leaving single germ detection behind and looking ahead to a fingerprinting of the whole bacterial community. With this unique microbial fingerprint, even forensic analyses could be made possible, as the composition of bacterial biofilm differs from person to person, whether sampled from the oral cavity (Aas et al., 2005) or the skin (Fierer et al., 2010). The oral microbiome displays a large variability; various microhabitats like gingival tissue, tongue, saliva, supra- or subgingival locations facilitate biofilm formation and growth already at early ages (Papaioannou et al., 2009). Keijser et al. (2008) showed that the vast majority (namely 99.6\%) of sequences in saliva and subgingival plaque samples of adults belong to one of the seven major phyla: Actinobacteria, Bacteroides, Firmicutes, Fusobacteria, Proteobacteria, Spirochetes, or candidate division TM7. Lazarevic et al. (2010) could prove these findings in salivary samples. However, not only bacterial phyla can be tagged; these new methods can show bacterial diversity on all taxonomic levels from the phylum through the genus level. This identification of bacteria takes place over nine hypervariable regions (V1 through V9) of the 16S rRNA gene used to distinguish thousands of species sequences of one sample from another (Chakravorty et al., 2007; Huse et al., 2008). The huge amount of sequence data gained with these methods puts common knowledge of pathogens into a new perspective. Many bacteria previously known to be pathogens were now also found in healthy subjects. Certain bacterial species like Streptococci or Acinetobacter were more related to health while other like Treponema, Fusobacteria, and Prevotella were associated with oral disease states in adults (Ledder et al., 2007; Abusleme et al., 2013; Wade, 2013). At the same time, hundreds of rare bacteria have been neglected in analysis which may be due to their being difficult to cultivate and/or detect, or because their detected numbers do not allow for statistical analysis. Focusing on single species can lead to distortion of the real picture of disease. But, how can we compare patients, possible treatment effects, sampling methods, etc. when the information we get consists not only of 20 bacteria but of thousands of species? In addition, how can clinicians translate this information? In this work, we test and show the exemplary comparison of two subgingival biofilm sampling modes for 454-pyrosequencing. We hypothesize that a modification of the clinical sampling mode can lead to a difference in the microbiome composition. We discuss statistical analyses and bioinformatics to provide information on how to compare on an inter- and intra-individual level the microbiota of the subgingival biofilm of healthy children. Issues related to small sample sizes and sample size calculation are also addressed. The overarching aim of this study is to reach the community of dentists and orthodontists with yet scarce knowledge of the potential of microbiome studies. We wish to raise interdisciplinary awareness for the clinical perspective of oral microbiome research in view of translational medicine from bench-side to patient. According to this announcement we, firstly, address the influence of external factors (i.e., clinical sampling methods) on the stability of a microbiome and, secondly, aim to support methods, possibilities and approaches to change and control the subgingival microbiome in human disease through our clinical work and toward a standardized pipeline. Finally, we look at interdisciplinary collaborations to facilitate the transfer of oral microbiome data to real clinical application.

\section{MATERIALS AND METHODS}

\section{Subjects}

For this comparative study, we included five ten-year-old children of both sexes (two male, three female). All recruited children had fulfilled the following criteria for participation in this study: late mixed dentition with first premolars fully erupted in the upper arch, good general and periodontal health, no bleeding on probing, a plaque index below $30 \%$, no antibiotic intake within the previous 3 months, and no use of antiplaque solutions. Prior to enrollment, written informed consent was obtained from each participant and one of his or her parents. The study was approved by the institutional review board at the Medical University of Graz. Written consent was also obtained explicitly for the publication of the intraoral photo in Figure 1.

\section{Sampling of Subgingival Biofilm: Modes A and B}

Clinical examination and sampling was performed by a single, experienced investigator. Sampling was always performed after 


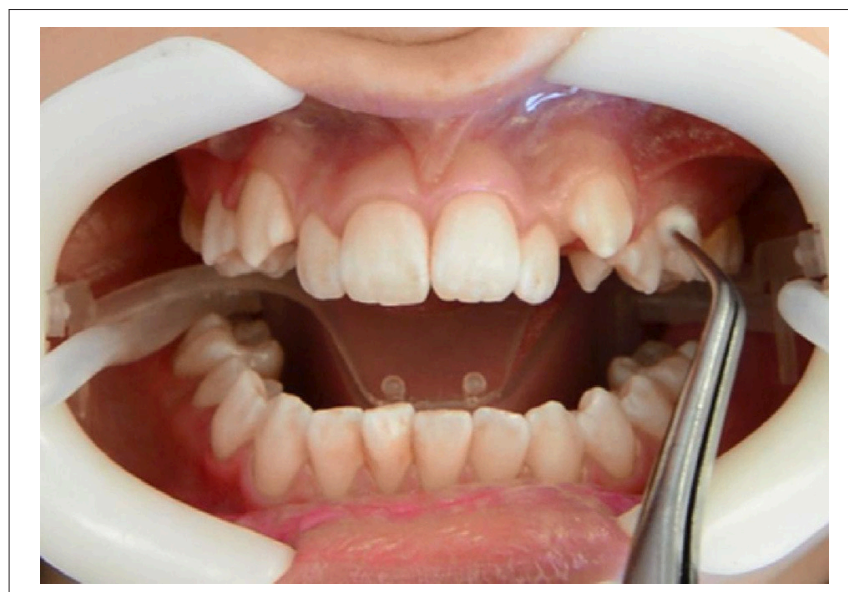

FIGURE 1 | Sampling in the maxilla after full arch isolation.

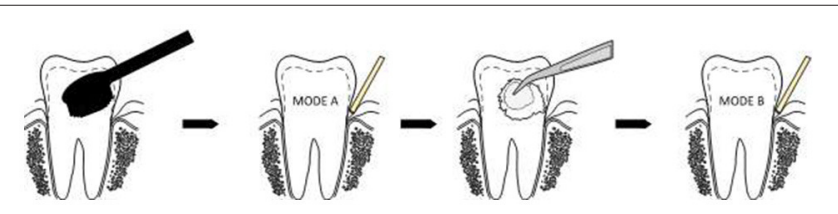

FIGURE 2 | Subgingival paper point sampling before (Mode A) and after (Mode B) supragingival cleansing with a sterile cotton pellet.

standardized oral hygiene instructions over a period of 3 weeks. Prior to subgingival sampling, supragingival plaqueas disclosed by an indicator-was removed with a previously unused toothbrush and water performed by the children themselves. No toothpaste was used. Full arch isolation in the maxilla was obtained by NOLA Dry Field (c) system as shown in Figure 1. Subgingival biofilm was then collected from the upper first premolars and first molars. Biofilm sampling had to be performed very carefully, so as not to traumatize the young gingival tissue in the absence of pockets commonly seen in periodontal disease. Healthy subjects and especially children have small compartments that make probing subgingival biofilm very challenging. Sterilized and UV-treated paper points (ISO15, Antaeos ${ }^{\circledR}$ ) were inserted into the subgingival sulcus parallel to the gingival margin at eight sites located mesio- and distobuccally of the four index teeth in two run-throughs differing slightly in their sampling mode. Sampling was done, firstly, excluding (Mode A) and, secondly, including (Mode B) supragingival cleansing with a sterile cotton pellet (see Figure 2). So the main difference between Mode A and Mode B refers to the supragingival cleaning. Samples were taken in sequence during the same sampling procedure from the same eight sites and then pooled and stored at $-20^{\circ} \mathrm{C}$ until processed (see Figure 3).

\section{DNA Extraction}

Bacterial DNA was prepared by first placing the paper points in a mixture of $380 \mu \mathrm{l}$ of MagNA Pure Bacteria Lysis Buffer (Roche Applied Science, Mannheim, Germany) and $20 \mu \mathrm{l}$ of proteinase $\mathrm{K}$ solution $(20 \mathrm{~g} / \mathrm{l})$. The suspension (including

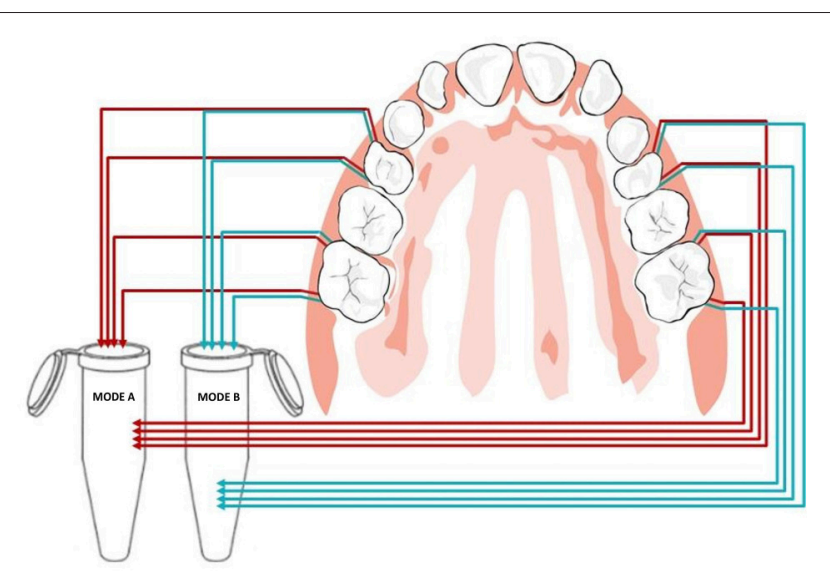

FIGURE 3 | Pooled paper point sampling of the gingival sulcus: paper points were inserted at eight sites before (Mode A) and after (Mode B) supragingival cleansing shown in red and blue, respectively.

the paper points) was incubated at $65^{\circ} \mathrm{C}$ for $10 \mathrm{~min}$ and subsequently at $95^{\circ} \mathrm{C}$ for another $10 \mathrm{~min}$. After removal of the paper points, the suspension was transferred into the MagNA Pure Compact Sample Tube (Roche Diagnostics, Mannheim, Germany). Automated DNA extraction was performed on the MagNA Pure Compact instrument (Roche) according to manufacturer instruction using the MagNA Pure Compact Nucleic Acid Isolation Kit I (Roche Diagnostics, Mannheim, Germany). Prior to the start of DNA extraction, the instrument adds the heterologous IC automatically. For extraction of bacterial DNA, the DNA Bacteria Purification protocol was used according to manufacturer instructions. DNA was eluted in $50 \mu \mathrm{l}$ $\mathrm{dH}_{2} \mathrm{O}$ and stored at $-20^{\circ} \mathrm{C}$ until use.

\section{4-Pyrosequencing}

Pyrosequencing was performed by DNAvision (avenue George Lemaitre 25B, 6041 Charleroi, Belgium, http://www.dnavision. com/). Microbial diversity was analyzed targeting 16S rRNA hypervariable regions V5 and V6. Pyrosequencing primers used are shown in Supplementary Table S1 containing 16s rRNA target specific primer sequences 784F-5'-AGAGTTTGA TCCTGGCTC-3' and 1061R-5' ATTACCGCGGCTGCTGG-3' (italic) according to Andersson et al. (2008), MID sequence (underlined), four bases key sequence and the Roche Titanium adaptor sequences (bold). For each sample, a PCR mix of 100 $\mu \mathrm{l}$ was prepared containing $1 \times$ PCR buffer, $2 \mathrm{U}$ of KAPA HiFi Hotstart polymerase and dNTPs (Kapa Biosystems), $300 \mathrm{nM}$ primers (Eurogentec, Liege, Belgium), and $60 \mathrm{ng}$ total DNA. Thermal cycling consisted of initial denaturation at $95^{\circ} \mathrm{C}$ for $5 \mathrm{~min}$, followed by 25 cycles of denaturation at $98^{\circ} \mathrm{C}$ for $20 \mathrm{~s}$, annealing at $56^{\circ} \mathrm{C}$ for $40 \mathrm{~s}$, and extension at $72^{\circ} \mathrm{C}$ for $20 \mathrm{~s}$, with a final extension of $5 \mathrm{~min}$ at $72^{\circ} \mathrm{C}$. Amplicons were visualized on 1\% agarose gels using GelGreen Nucleic Acid gel stain in 1xTAE (Biotium) and were cleaned using the Wizard SV Gel and PCR Clean-up System (Promega, Mannheim, Germany) according to manufacturer instructions. Amplicon DNA concentrations were determined using the Quant-iT 
PicoGreen dsDNA reagent and kit (Life Tech, Carlsbad, USA) following manufacturer instructions. After quantitation, cleaned amplicons were mixed in equimolar ratios into a single tube. The final pool was again purified using Agencourt Ampure $\mathrm{XP}$ purification systems according to manufacturer instructions (Agencourt Biosciences Corporation-Beckman Coulter, USA) and then eluted in $100 \mu \mathrm{l}$ of $1 x \mathrm{xTE}$. The concentration of the purified, pooled DNA was determined using the QuantiT PicoGreen dsDNA reagent and kit (Life Tech, Carlsbad, USA) following manufacturer instructions. Pyrosequencing of an equimolar pool of 10 samples on 1/8 PTP was carried out using primer A on a 454 Life Sciences Genome Sequencer FLX instrument (Roche, Mannheim, Germany) and following GS FLX Titanium Sequencing Kit XLR70 chemistry (Roche 454 Life Science, Branford, CT, USA) according to manufacturer instructions which resulted in 4131-19,943 raw reads per sample. Sequences are available at NCBI, accession number: SRP080750.

\section{Sequence Data Analysis}

In the first step, generated sequence data was assessed for quality. By using our own perl script only sequences with a minimum length of 150 bases, average Phred score of 25 and no ambiguous bases were selected for use in the downstream analysis. The remainder of the analysis was performed with the Quantitative Insights Into Microbial Ecology (QIIME) pipeline version 1.3.0 using standard parameters, including uclust (Edgar, 2010) for building OTUs with a similarity threshold of 0.97 , pyNAST (Caporaso et al., 2010) for representative sequence alignment, FastTree (Price et al., 2009) to generate the phylogenetic tree and RDP classifier (Wang et al., 2007) for taxonomic assignment. Chimeric sequences were removed using ChimeraSlayer with default QIIME settings after OTU picking and taxonomic assignment on aligned representative sequences.

Estimation of the within-samples diversity (alpha diversity) was performed with the Simpson (1949), Shannon (1948), and Chao (1984) metrics.

In the final step, we generated PCoA plots and performed hierarchical clustering analysis based on distance matrix from an unweighted UniFrac phylogenetic method (Lozupone et al., 2011) which enabled the between-samples comparison (beta diversity) of the microbial communities.

For the beta diversity analysis and normalization, sample heterogeneity was excluded by rarefication of all samples to the sample with the lowest number of reads.

\section{Statistical Analysis}

Statistical analyses were performed using SPSS version 22.0 (SPSS Inc., Chicago, IL), $\mathrm{R}$ version 3.11 ( $\mathrm{R}$ Core Team, 2015) and PASS 2012 (NCSS, LLC. Kaysville, Utah). Data are presented as median and as interquartile range (lower quartile 25-percentile and upper quartile 75-percentile). Inter-individual differences of the median relative abundances served for the comparison of the two sampling modes. Wilcoxon tests with Bonferroni correction for multiple testing were used to compare Mode A (excluding supragingival cleansing) and Mode B (including supragingival cleansing) on phylum level $(n=6)$, on class level $(n=14)$, on order level $(n=19)$, on family level $(n=27)$ and on genus level $(n=29)$. All names of the specific bacteriae are provided in Tables 2.1-2.5 and in Figure 6. Paired $t$-tests were performed additionally, since the small sample size did not allow to verify the assumption of normality for the data. All reported $p$-values were two-sided. After Bonferroni correction statistical significance was considered with $p<0.0083$ at the phylum level, $p<0.0036$ at the class level, $p<0.0026$ at the order level, $p<0.0019$ at the family level and $p<0.0017$ at the genus level.

To test differences in abundance for a total of $n$ taxa between two groups, the rank-sum test including multiple testing with Bonferroni correction was used to estimate the power and the sample size for different effect sizes for alpha level of $0.05 / \mathrm{n}$.

Significance for PCoA (beta-diversity) analyses was checked with multivariate permutation tests using the nonparametric method "Adonis" (999 permutations) included in the package "vegan" of the QIIME-incorporated version of "R."

\section{RESULTS}

\section{Pyrosequencing and Diversity Indices}

A total of 92,680 sequences were derived from pooled DNA of 10 samples in the pyrosequencing assay. Data filtering and quality control resulted in 67,218 sequences with an average sequence length of 243 bp (SD 6.52; range 231-255), read numbers per sample ranging from 2937 to 14,629 sequences.

Rarefaction curve analysis showed that the sequencing effort was not sufficient to cover the whole microbiota in the analyzed samples. It is very likely that rare taxa and taxa with low abundances have been missed (Supplementary Figure S1). Nevertheless, this should not significantly influence results, since low abundant taxa do not shift the complete microbiota profiles and the tools used for their comparison are robust enough to compensate for low deviances.

The number of OTUs defined at $97 \%$ identity ranged from 532 to 1107 (as shown in Table 1). Sample richness, which in this analysis equals to the number of OTUs, as well as sample diversity (Shannon Index range 4.26-5.31) did not demonstrate major differences between the two sampling modes.

\section{Taxonomic Summary}

Analysis over the whole microbiota showed a predomination of the five main phyla: Actinobacteria (2.8-24.6\%), Bacteroidetes (9.2-25.1\%), Proteobacteria (4.9-50.6\%), Firmicutes (16.5-57.4\%) and Fusobacteria (2.2-17.1\%) (Figure 4).

The median relative abundances for all representatives in the profiled microbiomes on different taxonomic levels (phylum, class, order, family and genus) are given in Table 2.

Figures 4, 5 show sampling modification effects on relative abundances on phylum level (barchart) and on class level (heat map). A concordant qualitative pattern within individuals and differences between individuals could be shown regardless of the sampling mode.

\section{Statistical Analyses of Sampling Modes and Sample Size Calculation} Differences between Sampling Modes A and B

Effects in the subgingival microbiome profiles possibly due to sampling modification are displayed by area graphs in Figure 6. $P$-values from Wilcoxon signed rank tests and the median 
TABLE 1 | Sequencing information and diversity estimates for the subgingival microbiome profiles in five healthy children before (Mode A) and after (Mode B) supragingival cleansing.

\begin{tabular}{|c|c|c|c|c|c|c|c|c|c|c|c|}
\hline PatID & nReads & IAvg & $\begin{array}{l}\text { nReads after } \\
\text { trimming }\end{array}$ & $\begin{array}{l}\text { richness } \\
\text { (nOTUs) }\end{array}$ & Evenness & Shannon & Simpson & S.chao1 & se.chao1 & S.ACE & se.ACE \\
\hline $1 \mathrm{~A}$ & 9628 & 248.56 & 7298 & 758 & 0.70 & 4.61 & 0.96 & 1359 & 86.95 & 1358 & 22.22 \\
\hline $2 \mathrm{~A}$ & 6866 & 249.21 & 5086 & 621 & 0.72 & 4.65 & 0.97 & 1171 & 85.29 & 1259 & 23.53 \\
\hline $3 A$ & 4131 & 239.85 & 2937 & 599 & 0.83 & 5.31 & 0.99 & 1443 & 138.83 & 1385 & 23.33 \\
\hline $4 \mathrm{~A}$ & 19,943 & 241.53 & 14,629 & 1099 & 0.68 & 4.77 & 0.97 & 1827 & 88.98 & 1899 & 26.73 \\
\hline \multirow[t]{4}{*}{$5 A$} & 6193 & 238.53 & 4425 & 662 & 0.79 & 5.14 & 0.98 & 1174 & 77.59 & 1239 & 22.17 \\
\hline & & & avg & 748 & 0.74 & 4.90 & 0.97 & 1395 & & 1428 & \\
\hline & & & $\min$ & 599 & 0.68 & 4.61 & 0.96 & 1171 & & 1239 & \\
\hline & & & $\max$ & 1099 & 0.83 & 5.31 & 0.99 & 1827 & & 1899 & \\
\hline $1 \mathrm{~B}$ & 8817 & 245.08 & 6497 & 887 & 0.75 & 5.12 & 0.98 & 1592 & 91.60 & 1679 & 25.74 \\
\hline $2 \mathrm{~B}$ & 4987 & 254.8 & 3861 & 532 & 0.72 & 4.53 & 0.95 & 1053 & 87.21 & 1162 & 22.73 \\
\hline 3B & 11,711 & 240.16 & 8348 & 786 & 0.64 & 4.26 & 0.94 & 1329 & 77.56 & 1376 & 22.12 \\
\hline $4 \mathrm{~B}$ & 14,979 & 231.59 & 10,166 & 1107 & 0.68 & 4.75 & 0.96 & 1796 & 81.13 & 1938 & 27.41 \\
\hline \multirow[t]{4}{*}{$5 B$} & 5425 & 242.6 & 3971 & 546 & 0.73 & 4.60 & 0.97 & 1042 & 82.73 & 1093 & 20.61 \\
\hline & & & avg & 772 & 0.70 & 4.65 & 0.96 & 1362 & & 1450 & \\
\hline & & & $\min$ & 532 & 0.64 & 4.26 & 0.94 & 1042 & & 1093 & \\
\hline & & & $\max$ & 1107 & 0.75 & 5.12 & 0.98 & 1796 & & 1938 & \\
\hline
\end{tabular}

Filtered sequence summary

Sequence Alphabet Filter

Primer trimmer

$\mathrm{N}$ count $>0$ seq filter

GreaterThan 150 sequence length filter 22,574

Exponential Quality Filter

Total numbers of OTUs similarity 97\% 5601

relative abundance at all five taxonomic levels were used to display differences between Mode A (excluding supragingival cleansing) and Mode B (including supragingival cleansing) for all bacterial species on all levels (Tables 2.1-2.5). Nearly statistically significant differences $(p=0.063)$ between sampling Modes A and $\mathrm{B}$ could be shown for the phylum of Bacteroidetes based on Wilcoxon signed-rank tests: Bacteroidia (class), Bacteroidales (order), Prevotellaceae (family), and Prevotella (genus). The latter was shown to be statistically significant $(p=0.047)$ when the paired $t$-test was applied. Paired $t$-tests were assessed additionally to Wilcoxon signed-rank tests due to the small sample size in the study so as to prove that nearly statistically significant results with Wilcoxon signed-rank tests become significant. In general, the Wilcoxon signed-rank test cannot be significant for a sample size smaller than 6, for two sided testing. For one sided testing, a sample size of at least 5 is needed for the result to be significant. For the paired $t$-test there is no such limitation. Notably, after correction for multiple testing, almost all differences were no longer nearly significant (Table 2.1 through Table 2.5 and Figure 6).

\section{Sample Size}

Based on the Wilcoxon signed rank test and the assumption of a power of $85 \%$ (as required by the local Ethics Committee), a high variety of different sample sizes are thus needed for the bacterial representatives on different taxonomic levels. The bigger the effect size and the smaller the standard deviations, the fewer samples are needed. For example Table 2.3 shows that the calculated sample sizes needed for the 19 bacterial species on order level ranged between 8 for Bacteroidales (median $A=3.7$; median $B=7.9$ ) and 82,194 for Actinomycetales (median $A=7.9$; median $B=8.6$ ), despite the huge sample size of 110,445 needed for more or less rare and undefined representatives. At class level, only two more subjects for Bacteroidetes (phylum)-Bacteroidia and, at order level, only three more subjects for Bacteroidetes (phylum)-Bacteroidales would have been needed to reach a power of $85 \%$ and to obtain a significant result for the Wilcoxon signed rank test, assuming that effect size and standard deviation remain constant (see Table 2).

\section{Multivariate Analysis: Principal Coordinate Analysis (PCoA) and Hierarchical Clustering}

Principal coordinate analysis ( $\mathrm{PCoA}$ ) on distance matrices calculated with unweighted UniFrac showed a grouping of the paired samples (Figure 7). Pairs, shown in the same color, are close together in all three dimensions, except for Sample 


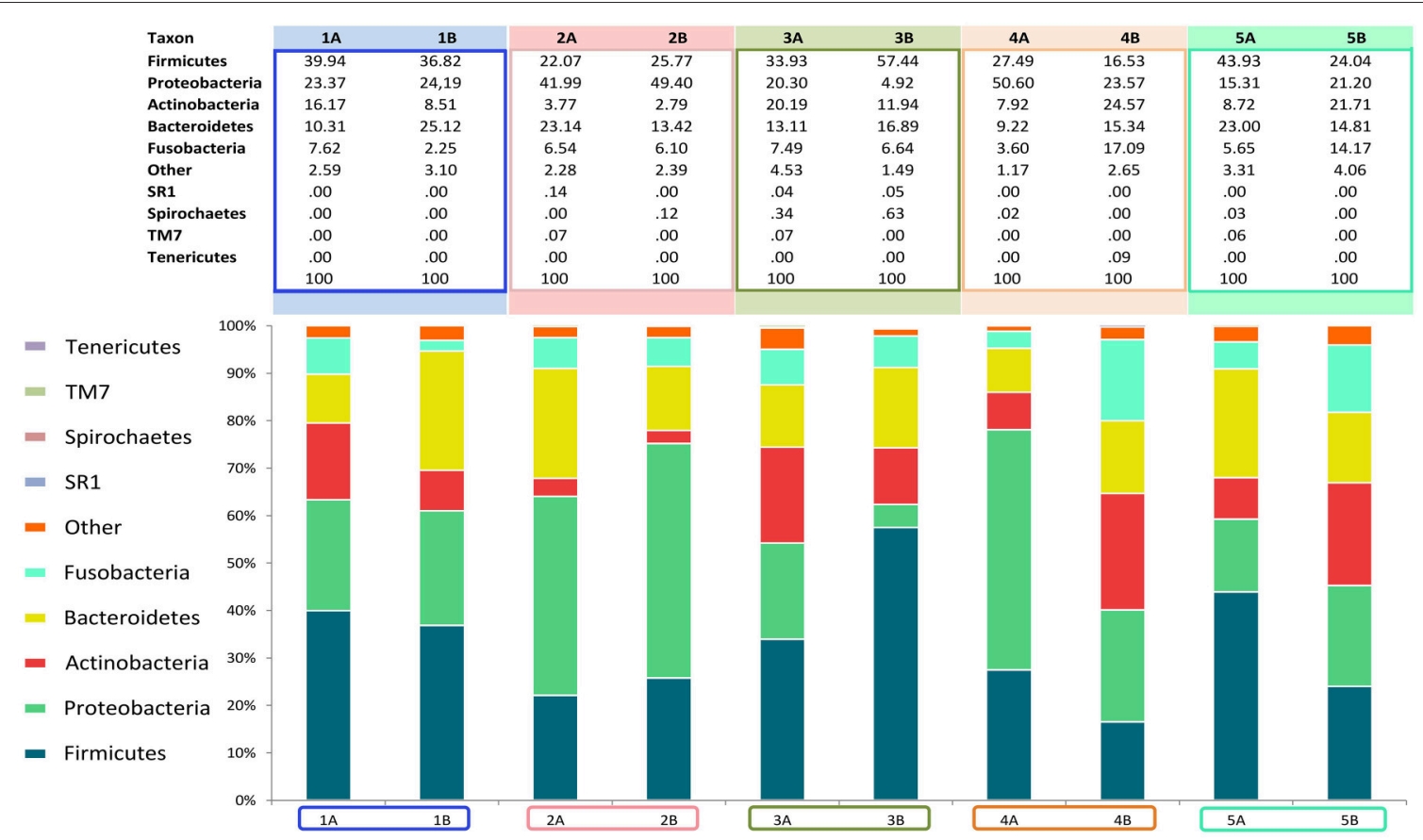

FIGURE 4 | Relative abundance of all phyla identified in the subgingival microbiome of 5 healthy children before (Mode A) and after (Mode B) supragingival cleansing.

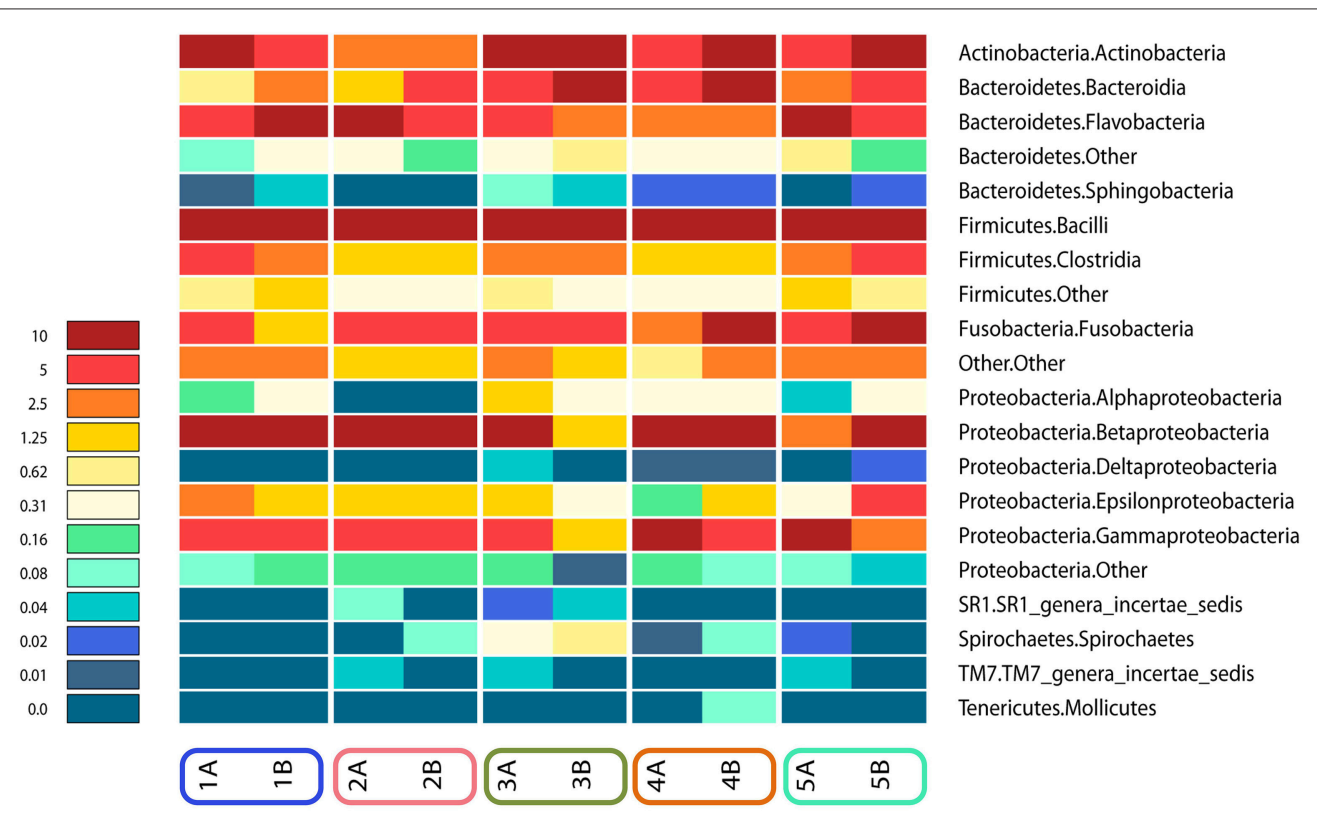

FIGURE 5 | Heat map of relative abundances of all classes identified in the subgingival microbiome of 5 healthy children before (Mode A) and after (Mode B) supragingival cleansing.

$5 \mathrm{~A}$ that shows a respective deviation and shows similarity with both samples from Subject 1. The results using Adonis (Permutational MANOVA) revealed no grouping of the samples according to Mode A or Mode B ( $p=0.914$ by $\left.R^{2}=0.09\right)$ and thus no significant effects between Mode
A and Mode B. PCoA results showed greater variability BETWEEN than WITHIN individuals. This observation was also supported by agglomerative hierarchical clustering analysis with average linkage on unweighted UniFrac distance (Figure 8). 

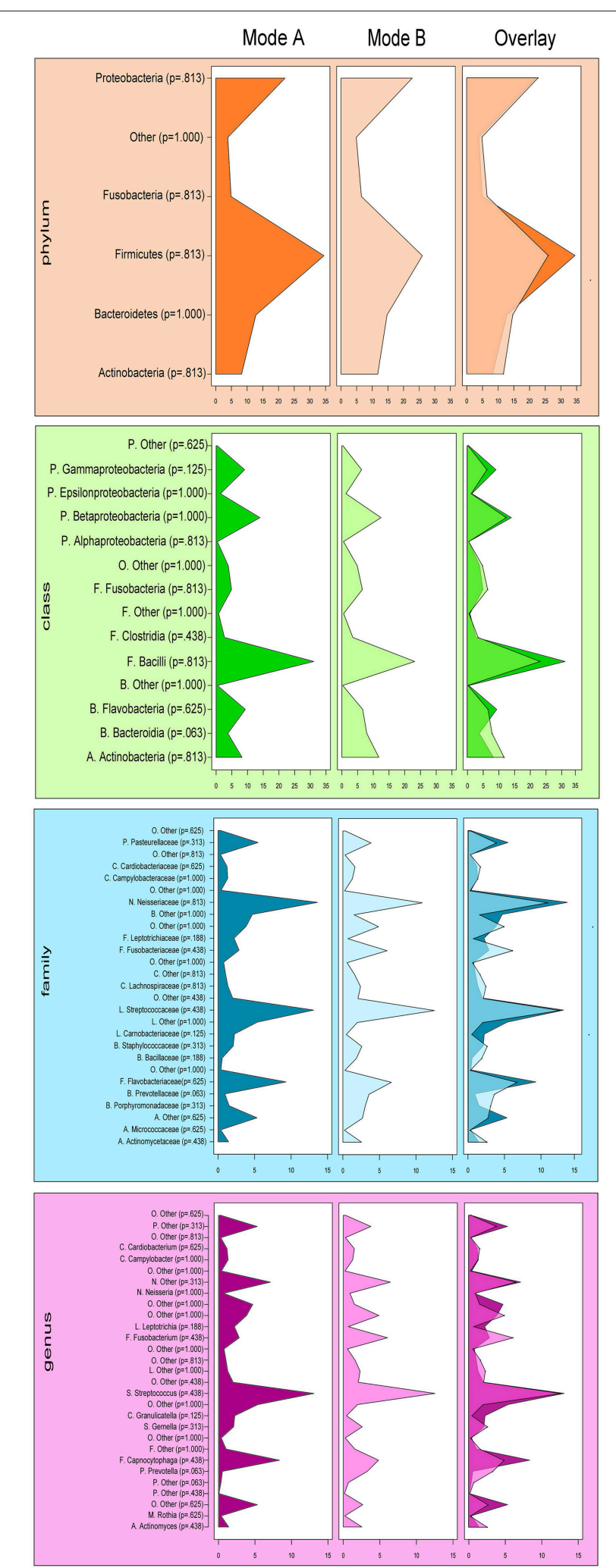

FIGURE 6 | Comparison of the median relative abundance of bacterial species present in all samples before (Mode A) and after (Mode B) supragingival cleansing (phylum, class, family and genus taxon).

\section{DISCUSSION}

Oral microbiota are considered one of the main risk factors for periodontal diseases affecting up to $90 \%$ of the world population (Pihlstrom et al., 2005). Oral biofilms have become increasingly important as a source of caries and periodontal disease as well as other bacterial infections in the human organism (Benítez-Páez et al., 2014). Some studies reveal evidence that oral pathogens play a role in various inflammatory diseases (Offenbacher et al., 2008). Few studies have deeply analyzed the composition of subgingival biofilm and elucidated the phylotypes/species associated with health or disease (Paster et al., 2001; Socransky and Haffajee, 2005; Ledder et al., 2007; Diaz, 2012; Abusleme et al., 2013).

The presented study analyzed using 454-pyrosequencing the data of five healthy 10-year-old children whose subgingival biofilm was examined excluding and including supragingival cleansing (Mode A and Mode B, respectively). The study aimed at assessing the effect of a slight modification of the clinical sampling technique for its accuracy in reflecting subgingival microbiome sequence data.

Retrieving adequate and reproducible samples is a challenge but awareness of the natural variability within subgingival microprints would enable us to distinguish pathological patterns at an early stage of disease. Corresponding in vivo conditions can best be studied in healthy children as shown in previous studies.

However, very few oral microbiome studies in healthy children have been performed so far (Papaioannou et al., 2009; Xin et al., 2013), some including pyrosequencing (Crielaard et al., 2011; Stahringer et al., 2012; Ling et al., 2013; Lif Holgerson et al., 2015). The study design of Crielaard et al. differs from ours in that they investigated microbial profiles of saliva collected from caries-diseased Dutch children aged 3-18 years. The biggest difference in the comparable age strata was the relative abundance of Firmicutes at 58\% in the saliva group and at 30\% in our subgingival samples, while the latter presented a higher proportion of Proteobacteria (22 vs. 12\%) and Fusobacteria (6 vs. $2 \%)$. Ling et al. used parallel barcoded 454-pyrosequencing to study the diversity and richness of salivary bacteria in 10 healthy children and adults. The bacterial diversity was found to be more complex in children than in adults (Ling et al., 2013) which could be interpreted as evidence for the relationship between biodiversity and health. In their sample comprising 60 children aged 3-6, the eight predominant phyla in supragingival plaque and saliva were present in proportions that were comparable to our study: $23-42 \%$ Firmicutes and 16-37\% Bacteroides (Ling et al., 2010). In a longitudinal study, Holgerson et al. looked at the oral microbiota of 207 Swedish babies at the age of 3 months and again at 3 years. The pyrosequencing data referred to 11 children with and 11 without caries. A significant increase in species richness and taxa diversity was described. Several taxa within the oral biofilms of the 3-year-olds could be linked to the presence or absence of caries. However, quantitative comparisons of the oral microbiota of children are possible only to a limited extent, since the investigators dedicated work differs in parameters such as study population (age, country, caries 
TABLE 2.1 | Comparison of the median relative abundance corresponding to 6 bacterial species on phylum taxon present in all samples before (Mode A) and after (Mode B) supragingival cleansing: median and IQR, $p$-values for paired $t$-test and Wilcoxon signed-rank test and sample size calculation.

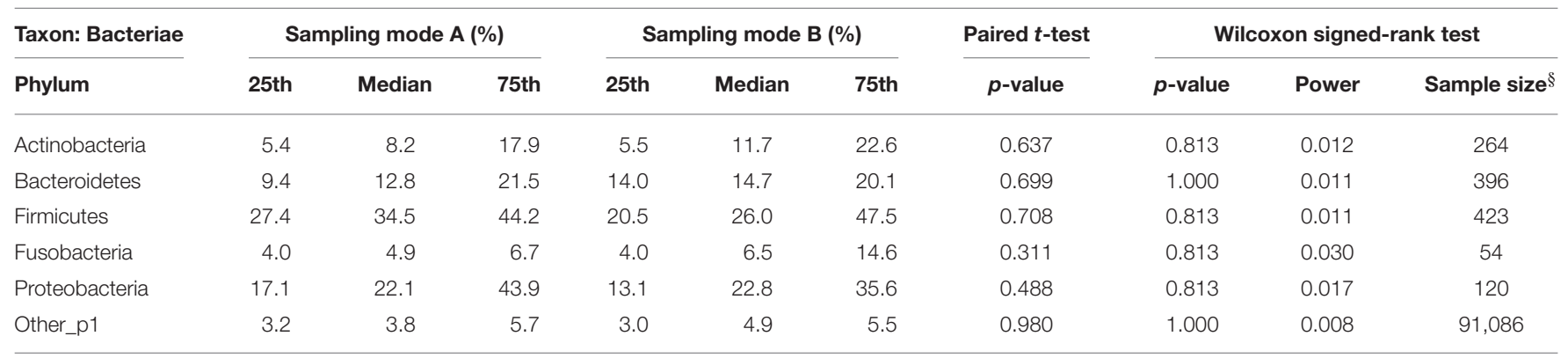

$\S$ Power of 0.85 is assumed.

After Bonferroni correction $p<0.0083$ is significant.

TABLE 2.2 | Comparison of the median relative abundance corresponding to 14 bacterial species on class taxon present in all samples before (Mode A) and after (Mode B) supragingival cleansing: median and IQR, $p$-values for paired $t$-test and Wilcoxon signed-rank test and sample size calculation.

\begin{tabular}{|c|c|c|c|c|c|c|c|c|c|c|}
\hline \multirow{2}{*}{$\begin{array}{l}\text { Taxon:Bacteriae } \\
\text { Phylum/Class }\end{array}$} & \multicolumn{3}{|c|}{ Sampling mode A (\%) } & \multicolumn{3}{|c|}{ Sampling mode B (\%) } & \multirow{2}{*}{$\frac{\text { Paired } t \text {-test }}{p \text {-value }}$} & \multicolumn{3}{|c|}{ Wilcoxon signed-rank test } \\
\hline & 25th & Median & 75th & 25th & Median & 75th & & $p$-value & Power & Sample size ${ }^{\S}$ \\
\hline \multicolumn{11}{|l|}{ ACTINOBACTERIA } \\
\hline Actinobacteria & 5.4 & 8.2 & 17.9 & 5.5 & 11.7 & 22.6 & 0.637 & 0.813 & 0.005 & 304 \\
\hline \multicolumn{11}{|l|}{ BACTEROIDETES } \\
\hline Bacteroidia & 1.6 & 3.7 & 5.1 & 4.7 & 7.9 & 11.9 & 0.006 & 0.063 & 0.428 & 7 \\
\hline Flavobacteria & 5.1 & 9.3 & 17.9 & 3.0 & 6.6 & 13.7 & 0.485 & 0.625 & 0.008 & 137 \\
\hline Other_c1 & 0.2 & 0.4 & 0.6 & 0.2 & 0.3 & 0.6 & 0.817 & 1.000 & 0.004 & 1278 \\
\hline \multicolumn{11}{|l|}{ FIRMICUTES } \\
\hline Bacilli & 25.3 & 31.1 & 38.0 & 16.1 & 23.2 & 42.6 & 0.673 & 0.813 & 0.005 & 381 \\
\hline Clostridia & 1.7 & 26.5 & 5.0 & 2.1 & 3.5 & 5.0 & 0.617 & 0.438 & 0.006 & 271 \\
\hline Other_c2 & 0.4 & 0.8 & 1.2 & 0.5 & 0.6 & 1.0 & 0.774 & 1.000 & 0.004 & 831 \\
\hline \multicolumn{11}{|l|}{ FUSOBACTERIA } \\
\hline Fusobacteria & 4.0 & 4.9 & 6.7 & 4.0 & 6.5 & 14.6 & 0.311 & 0.813 & 0.014 & 63 \\
\hline \multicolumn{11}{|l|}{ OTHER } \\
\hline Other_c3 & 3.2 & 3.8 & 5.7 & 3.0 & 4.9 & 5.5 & 0.980 & 1.000 & 0.004 & 105,044 \\
\hline \multicolumn{11}{|l|}{ PROTEOBACTERIA } \\
\hline Alphaproteobacteria & 0.1 & 0.4 & 1.1 & 0.2 & 0.5 & 0.6 & 0.714 & 0.813 & 0.005 & 507 \\
\hline Betaproteobacteria & 6.7 & 14.0 & 26.7 & 7.2 & 12.4 & 27.1 & 0.991 & 1.000 & 0.004 & 555,484 \\
\hline Epsilonproteobacteria & 0.3 & 1.3 & 2.4 & 0.8 & 1.3 & 3.7 & 0.587 & 1.000 & 0.006 & 229 \\
\hline Gammaproteobacteria & 5.3 & 9.1 & 16.0 & 2.4 & 6.3 & 7.0 & 0.129 & 0.125 & 0.038 & 26 \\
\hline Other_c4 & 0.3 & 0.3 & 0.6 & 0.1 & 0.3 & 0.6 & 0.491 & 0.625 & 0.008 & 140 \\
\hline
\end{tabular}

$\S$ Power of 0.85 is assumed.

After Bonferroni correction $p<0.0036$ is significant.

status), sampling sites (saliva, mucosal, and supragingival plaque) and molecular methods (DNA-DNA checkerboard, micro arrays, pyrosequencing). Papaioannou et al., for example, looked at five different oral habitats (saliva, tongue, soft tissue, subgingival, and total supragingival plaque) of 93 children from three different age groups using whole genomic probes for 38 species and the checkerboard DNA-DNA hybridization technique. The authors suggest a gradual maturation of the oral microbiota in children displaying patterns of colonization similar to those seen in adults (Papaioannou et al., 2009). However, until now most studies have analyzed salivary biofilm, as it is easier to sample (Luo et al., 2012; Stahringer et al., 2012; Ling et al., 2013;
Gomar-Vercher et al., 2014). Interestingly, Luo et al. who studied PCR-amplified bacterial DNA from the saliva of 20 children with caries and of 30 healthy ones found higher microbial diversity in samples from diseased oral cavities. In contrast to these findings, Gomar-Vercher et al. used pyrosequencing to analyze 110 saliva samples from children split into six groups according to caries severity and found the bacterial diversity to decrease with progressing disease. At the same time, intra-group differences were considerable (Gomar-Vercher et al., 2014). Stahringer and colleagues presented a longitudinal survey of salivary microbiota from twins using PCR amplification and 454 pyrosequencing of the $16 \mathrm{~S}$ rDNA hypervariable regions V1 and V2. Their findings 
TABLE 2.3 | Comparison of the median relative abundance corresponding to 19 bacterial species on order taxon present in all samples before (Mode A) and after (Mode B) supragingival cleansing: median and IQR, $p$-values for paired $t$-test and Wilcoxon signed-rank test and sample size calculation.

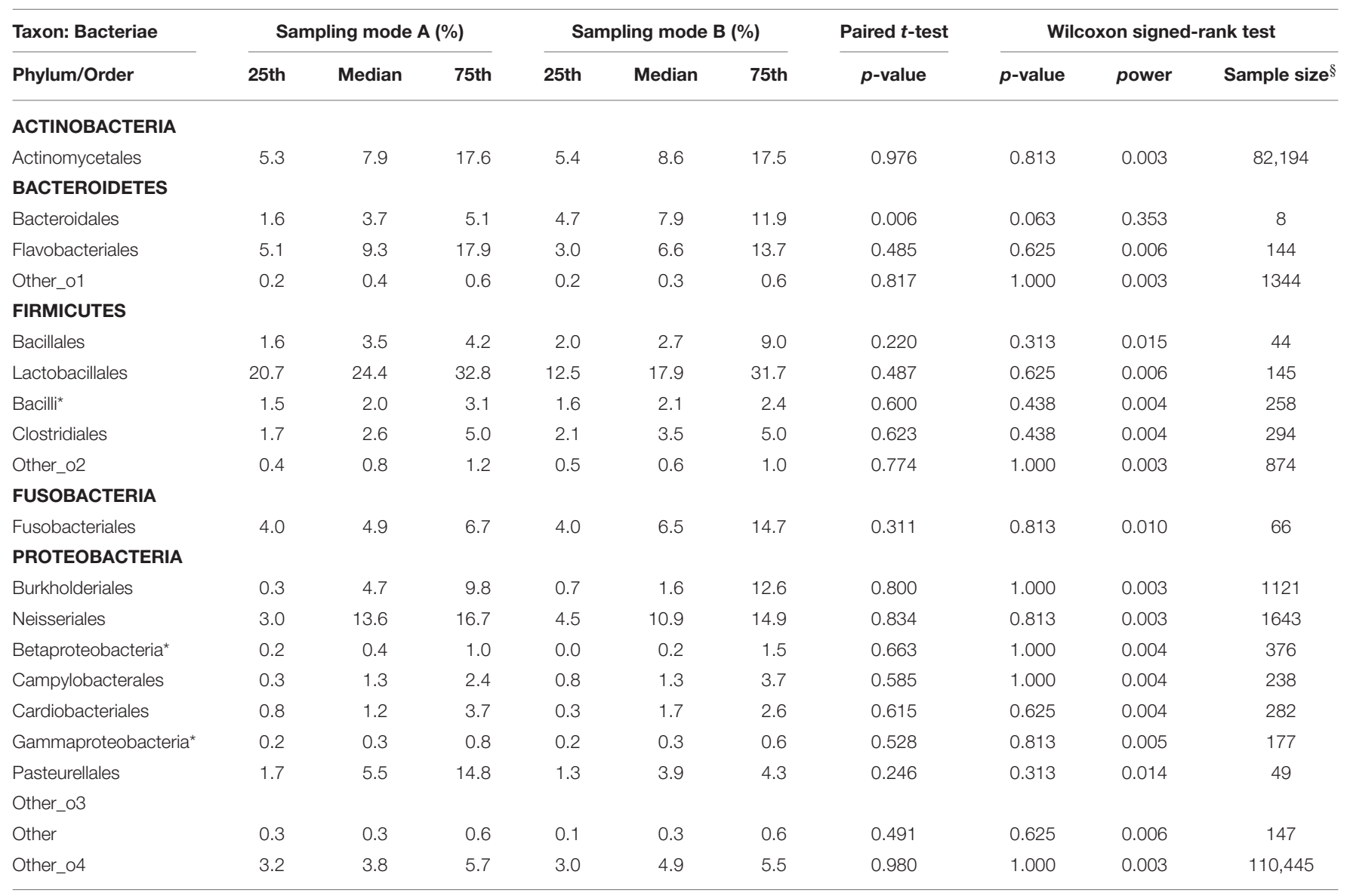

*Taxa marked with asterisk could not be assigned to any of the ordera and are shown on class level as lowest common taxon. ${ }^{\S}$ Power of 0.85 is assumed.

After Bonferroni correction $p<0.0026$ is significant.

point to the environment as the microbiome-determining factor showing greater differences between non-related subjects than within individuals or between twins (as long as they share a common habitat; Stahringer et al., 2012).

Standardized sampling procedures are a prerequisite for comparing subgingival microbiome data derived from research worldwide. The lack of heterogeneity and standardization for clinical protocols poses a limitation to data quality which should be noted by clinicians and microbiologists. In this context, we need to consider the diverse sampling methods reported for the collection of samples from a healthy oral cavity, not to mention the variability of pocket sampling in periodontally diseased patients. This can be illustrated by the example of just 10 published manuscripts dealing with the collection of samples from an intact oral cavity. They report using saline oral wash rinse (Ahn et al., 2011) or unstimulated whole saliva (Xin et al., 2013) for fluid collection; dental explorers (Xin et al., 2013), metal loops (Ling et al., 2010), metal curettes (Papaioannou et al., 2009) and wooden tooth picks (Keijser et al., 2008) for supragingival sampling; or wet and dry swabs and brushes (Aas et al., 2005; Papaioannou et al., 2009; Cortelli et al., 2012) and spatulas (Gohler et al., 2014) for mucosal sampling. Finally, subgingival sampling is currently being performed using either metal curettes (Papaioannou et al., 2009; Abusleme et al., 2013) or paper points (Cortelli et al., 2012; Griffen et al., 2012; Jünemann et al., 2012). For clinical and research purposes even exotic micropipettes or microelectrodes are used (Geibel, 2006). Potential sampling variability springs not only from the different instruments that can be utilized but also from processes taking place prior to sampling, such as plaque control, tooth cleaning, tooth isolation and drying, as well as from inadequate specifications regarding the sampling technique and time lines. Compared to standards that apply in other medical and laboratory settings, our clinical sampling is much like an elephant in a porcelain shop. Appropriate scientific input facilitates the development of a systematic and precise methodology which in turn can deliver reliable, high-quality clinical samples to the pipeline required in the field of molecular biology and medicine. Some authors have reported on the recovery of putative pathogens from paper point and curette sampling (Jervøe-Storm et al., 2007; Teles et al., 2008; Angelov et al., 2009; Sahl et al., 2014). Hartroth and colleagues have evaluated paper point sampling on bench (Hartroth et al., 1999), but these findings have yet to be tested under clinical conditions to establish the best practice. 
TABLE 2.4 | Comparison of the median relative abundance corresponding to 27 bacterial species on family taxon present in all samples before (Mode A) and after (Mode B) supragingival cleansing: median and IQR, $p$-values for paired $t$-test and Wilcoxon signed-rank test and sample size calculation.

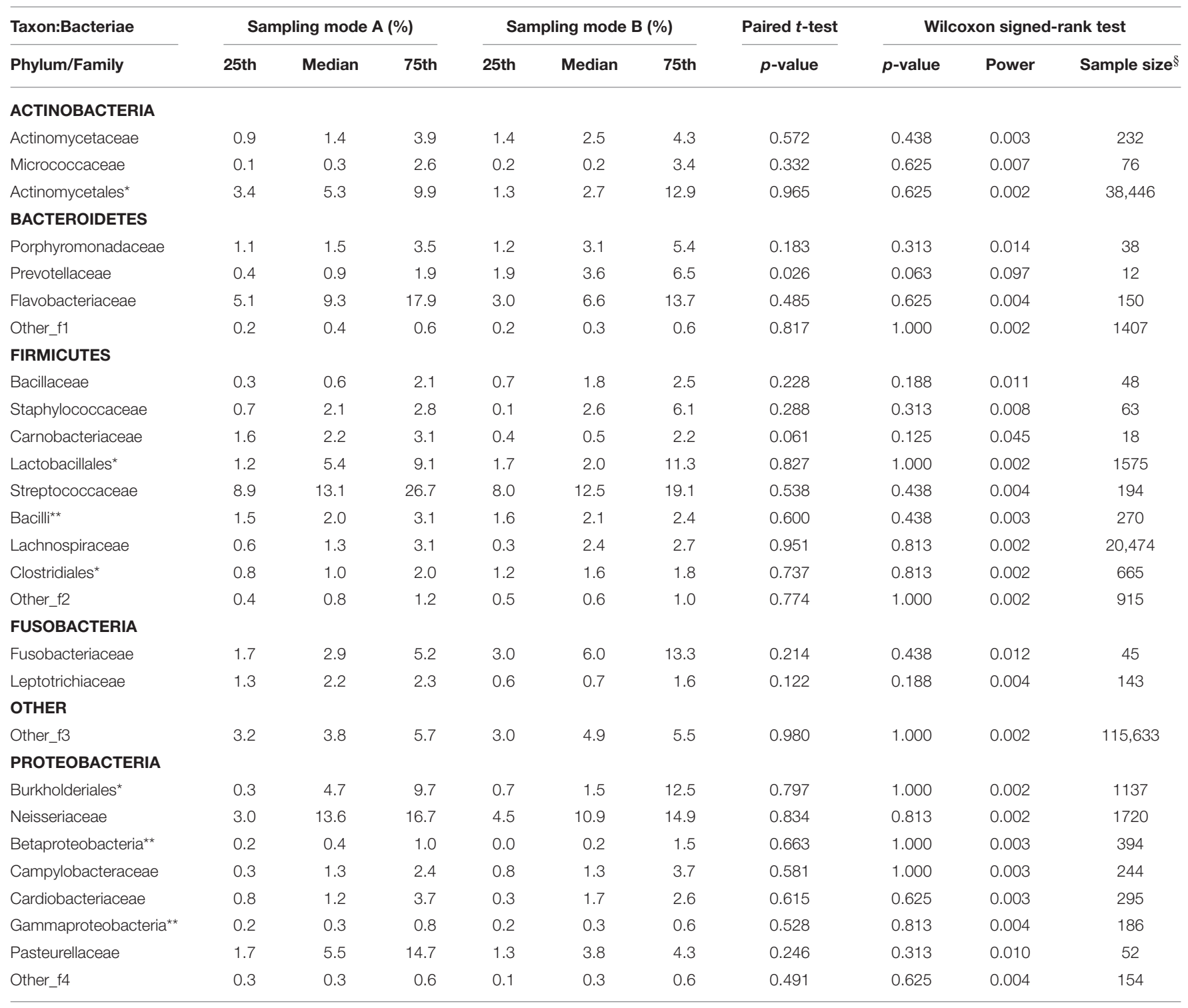

*Taxa marked with asterisk could not be assigned to any of the family taxon and are shown on ordera level as lowest common taxon.

**Taxa marked with asterisk could not be assigned to any of the family taxon and are shown on class level as lowest common taxon.

$\S$ Power of 0.85 is assumed.

After Bonferroni correction $p<0.0019$ is significant.

An aspect on which clinical researchers are in agreement is the removal of supragingival plaque before subgingival sampling. It is as obvious to them as taking off the shoes in the hallway before entering the living room. However, it is still debatable to what extent this cleansing should be performed to be efficient enough.

Generally, clinical sampling within the oral cavity of children can be tricky and calls for an experienced investigator. The clinical method in this study is designed around a younger study population with intact and tight subgingival compartments. The subgingival sulcus itself can best be imagined as an interface (of two millimeters) with a tight epithelium toward the periodontium but with a seamless junction (orifice) toward the supragingival surface. Thus, not only the removal of nonattached bacteria but also the microbial exchange between suband supragingival biofilm has to be taken into account in addition to the difficulty of precise sampling in this extremely limited subgingival space. Limited space makes sampling the subgingival sulcus of children a challenge. The deeper the sulcus, the more likely it is to strip supragingival biofilm before actually reaching the sulcus depth. In our case, sampling was performed by a single, experienced clinician excluding interrater variability. Paper points were gently slid parallel to the gingival margin in order to facilitate a painless and quick examination. This 
TABLE 2.5 | Comparison of the median relative abundance corresponding to 29 bacterial species on genus taxon present in all samples before (Mode A) and after (Mode B) supragingival cleansing: median and IQR, $p$-values for paired $t$-test and Wilcoxon signed-rank test and sample size calculation.

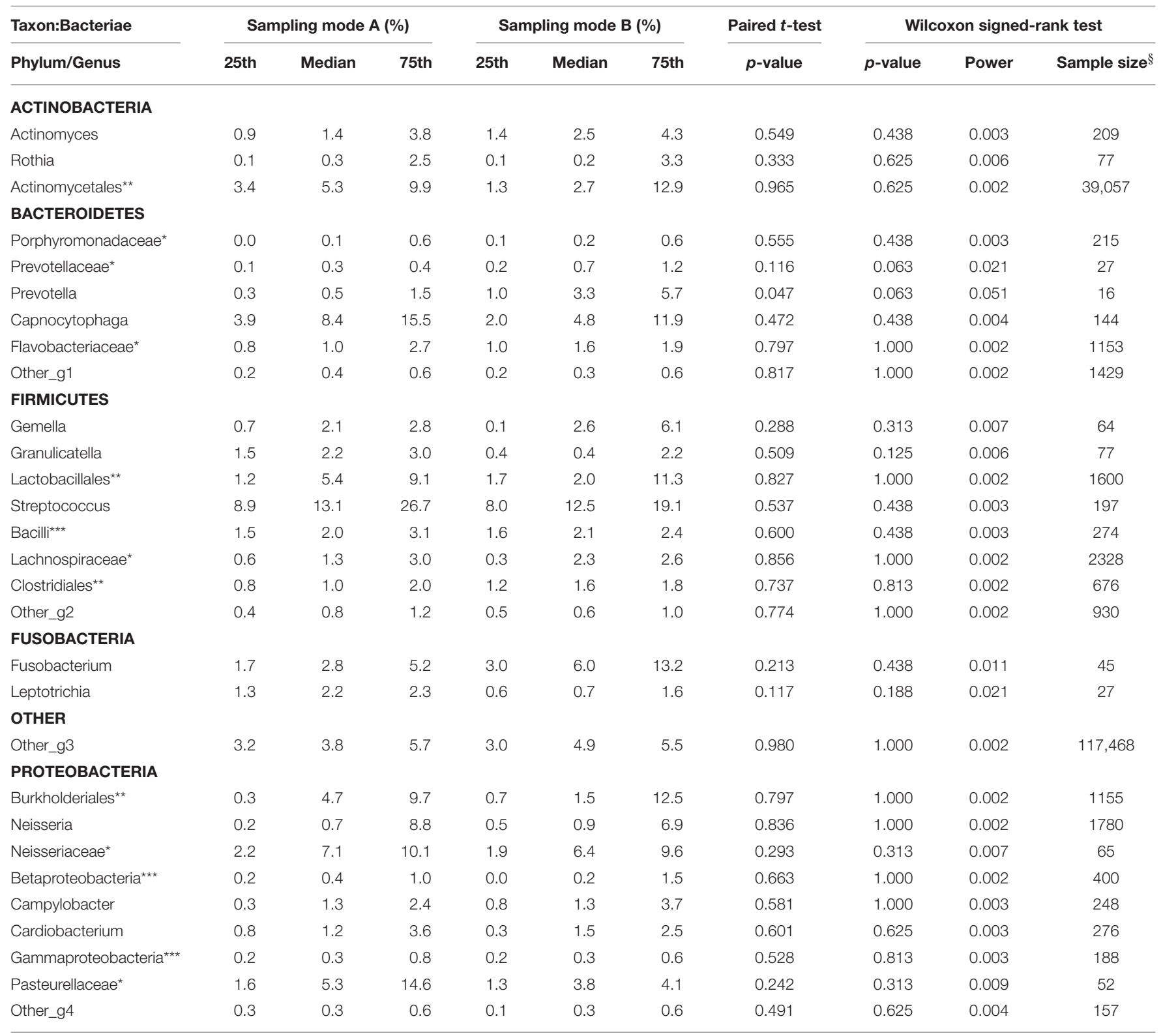

*Taxa marked with asterisk could not be assigned to any genera and are shown on family level as lowest common taxon.

${ }^{\star}$ Taxa marked with asterisk could not be assigned to any genera and are shown on order level as lowest common taxon.

${ }^{* * \star}$ Taxa marked with asterisk could not be assigned to any genera and are shown on class level as lowest common taxon.

$\S$ Power of 0.85 is assumed.

After Bonferroni correction $p<0.0017$ is significant.

contributes to better cooperation on behalf of the child and a short procedure prevents the paper point from becoming saturated with saliva. Paper points were used rather than the more invasive metal curette, as the latter could traumatize the subgingival sulcus and cause bleeding which was to be avoided at all costs. During the sampling procedure, the focus was placed on the drier subgingival areas of the upper arch, so as to optimize sample quality for DNA analysis. To ensure reproducibility, biofilm sampling followed a strict protocol (see also Methods above). Two modes (A and B) of the same sampling method were used for comparison. After supragingival cleaning using an electric toothbrush and water, sampling was performed, firstly, excluding (Mode A) and, secondly, including (Mode B) cleansing with sterile cotton pellets. The samples from a total of eight sites were pooled, so no inter-site comparisons were studied. Based on the paired samples, results of the PCoA intraindividual differences were relatively small despite the modest sample size in the present study. Also, permutational MANOVA showed no grouping of the samples according to Mode A or Mode B. It can be speculated that any existing deviation 

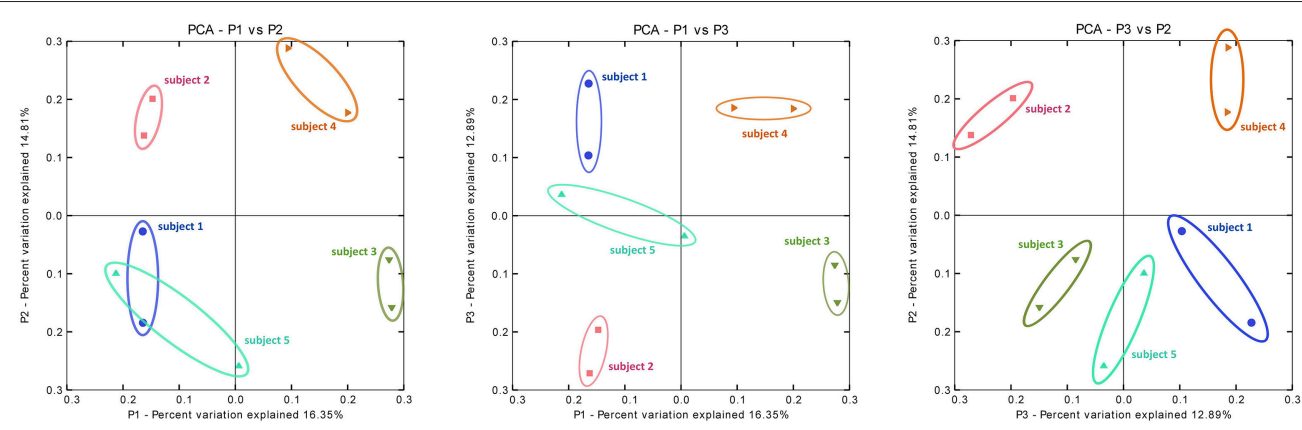

FIGURE 7 | Principal coordinate analysis (PCoA) on distance matrices calculated with unweighted UniFrac showing a grouping of the paired samples. PCoA plots of the subgingival microbiome profiles of five healthy children based on two run-throughs of paper point sampling. Colors and symbols represent one child. The results using Adonis (Permutational MANOVA) did not reveal grouping of the samples according to Mode A or Mode $\mathrm{B}\left(p=0.914\right.$ by $\left.R^{2}=0.09\right)$.

Cluster Dendrogram

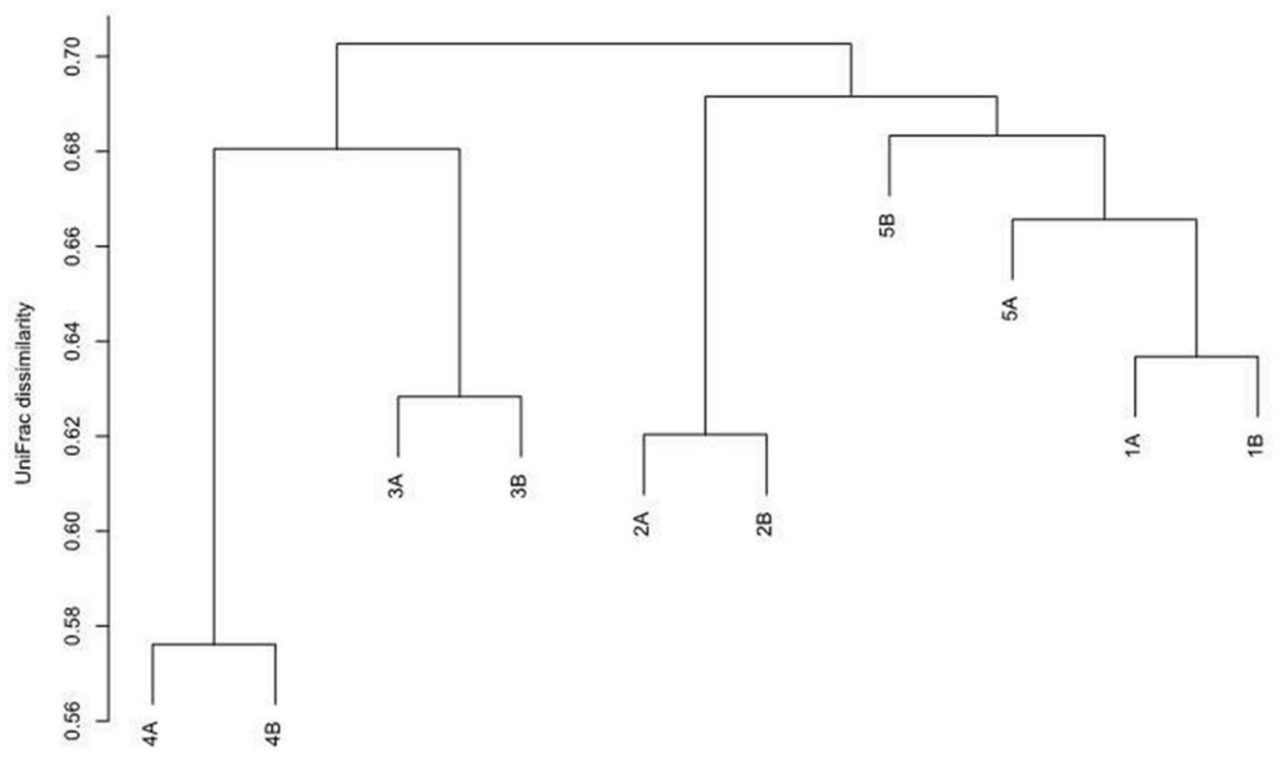

unweigh.unifrac.dm. dist hclust (", "average")

FIGURE 8 | Agglomerative hierarchical cluster analysis with average similarity based on unweighted UniFrac distances. The data employed are the rarefaction based normalized OTU abundances of five healthy children based on two run-throughs of paper point sampling with slight modification of the clinical sampling technique.

between the two sampling modes is very likely to correspond to a natural variation in oral biofilm of the individual subject and supragingival cleansing with a sterile cotton swab does not affect the composition of the subgingival biofilm of an individual. Importantly, it seems that there are no major effects due to the described sampling modification. However these "non-effects" between the two sampling modes refer to interindividual differences and obviously surpass the intra-individual "non-effects" which comes up to an overarching effect with relevance for future clinical studies
Analyses of the pooled DNA data using pyrosequencing is a timely and potentially interesting approach that also has numerous limitations. Table 1 shows that richness and evenness as well as Shannon diversity index do not indicate any differences in the above mentioned sampling modes (A and $\mathrm{B}$ ). However, it should be noted that the number of reads can influence the sensitivity of data; this issue is for example evident when comparing the quotient of the number of reads and richness for subjects $4 \mathrm{~A}$ and $5 \mathrm{~A}$. Such differences in the number of reads are practically unavoidable, therefore it is necessary to incorporate 
a normalization step into the data analysis which we did by rarefication of all samples to the sample with the lowest number of reads. Another option is the use of relative abundances as also applied in this study for statistical comparisons. The field under study here is so complex that it is impossible to ascertain at which exact point in the analysis problems occur, and whether the same amount of DNA was available from the participating children and/or if data loss had occurred even earlier. Laboratory workup is not discussed here in detail but the possibility of passive errors (e.g., during 16S RNA amplification for PCR) does exist despite standardized procedures. It also has to be remembered that this study looked at 16S rRNA hypervariable regions V5 and V6 only and not at the whole metagenome. This limitation also applies to other studies (Wu et al., 2010; Ahn et al., 2011; Griffen et al., 2011; Jünemann et al., 2012; Stahringer et al., 2012).

An asset of our study is the fact that (under the aforementioned conditions and for the afore-mentioned subjects) sample size calculations are presented for the bacterial species on all five levels as shown in Tables 2.1-2.5. Our small sample size poses a challenge for pyrosequencing and statistical testing, nevertheless different effects can be observed as visualized in Figures 6-8. In our study, sample sizes are part of the findings. Our work should emphasize that the challenge is the translation of sample size estimations to clinical feasibility. So far, statistically given sample sizes that would explain significantly and clinically relevant differences in the subgingival microbiome of children are neither practical nor ethical. Even a generous increase in samples, i.e. children, in our study would not have solved the problem. However, our data can serve as a pilot for future studies on the topic showing that large sample sizes are needed to elucidate microbial structures at different levels. The demanding task is to reflect the bacterial diversity as well as possible. However, as opposed to more common bacteria, rare species require huge sample sizes in order to unveil any significant differences. This task becomes even more complex with a higher number of rare species in a given sample. In order to study these issues, statistical methodology will have to be developed further. While appropriate technology is becoming increasingly available and affordable, sample sizes remain primarily a matter of practicality and ethics. Including healthy people, in particular children, or patients into clinical studies involves substantial costs for human resources and efforts beyond the daily routine for both sides: the study participants and the clinical staff. One way to practically increase sample sizes are standardized clinical protocols that would allow multi-site sampling in diverse populations.

In our analysis, the bacteria are only analyzed down to the genus level which is limiting. However, from the clinical perspective the data is noteworthy. Interestingly, the smallest calculated sample size roughly corresponds to the 20 bacteria available in commercial bacteria test kits applied in periodontology. However, some abundant bacteria are apparently not included in such test kits. In addition, numerous bacteria have not yet been identified and are assigned as "other" to superordinate taxonomic levels (see Tables 2.1-2.5). In this context, the limitation is the unattainable sample size for some phyla.
Another general issue that should be mentioned is the need for standardized protocols to facilitate the comparability of data generated in microbiome studies. Considerable interindividual differences in bacterial communities necessitate large samples. At the same time, intra-individual variability should also be considered in comparative studies. For microbiome data, new statistical methods like Adonis are needed and should be combined with methods from bioinformatics. For example, PCoA was used in our study to verify findings based on a small sample size, i.e., grouping of the paired samples for the within-comparison as intra-individual pairs clustered in all three dimensions. Importantly, many decisions regarding study design are made based on investigator experience (e.g., which distances to analyze with UniFrac). Future studies should aim at standardizing methodology to prevent bias and distortion of data.

Our work points at many challenges in the study of oral microbiomes. Our data, though based on a modest sample size, could serve as a reference for healthy children or may serve as a baseline for microbiome function in healthy individuals shedding new light on the frontiers of health and disease. The number of the species known is high (presently amounting to more than 600 taxa) and includes very rare ones whose role is yet unknown as well as other microbial representatives that are not bacteria (Moissl et al., 2002, 2003, 2005). Methods like DNA/RNA/metagenome sequencing need to be employed to begin to uncover the exact role of microbiota. Similarly, visualized analytics can give additional insight into individual species. However, we still need to learn which microbiota are imperative for the functioning of the whole. And we need to ask further questions: How does diversity make healthy? To what extent may individual health be attained by comparison with other individuals? The presented work employs modern approaches from several research areas but the focus remains on the clinical application and a contribution toward the standardization of procedures across all relevant disciplines.

\section{AUTHOR CONTRIBUTIONS}

ES: work conception and design; acquisition, analysis and interpretation of data; drafting and critical review of the manuscript; final approval of the work for publication. ST: analysis and interpretation of data; critical review of the manuscript; final approval of the work for publication. KE: analysis and interpretation of data; critical review of the manuscript; final approval of the work for publication. BK: study design; analysis and interpretation of data; draft and critical review of the manuscript; final approval of the work for publication.

\section{FUNDING}

This study was supported by the Hygiene Fund of the Institute of Hygiene, Microbiology and Environmental Medicine at the Medical University of Graz, Graz, Austria. 


\section{ACKNOWLEDGMENTS}

The authors are grateful to Michael Bozic (Laboratory at the Research Unit Molecular Diagnostics, Medical University of Graz) for his excellent technical assistance in DNA isolation.

\section{SUPPLEMENTARY MATERIAL}

The Supplementary Material for this article can be found online at: http://journal.frontiersin.org/article/10.3389/fmicb. 2016.02142/full\#supplementary-material

\section{REFERENCES}

Aas, J. A., Paster, B. J., Stokes, L. N., Olsen, I., and Dewhirst, F. E. (2005). Defining the normal bacterial flora of the oral cavity. J. Clin. Microbiol. 43, 5721-5732. doi: 10.1128/JCM.43.11.5721-5732.2005

Abusleme, L., Dupuy, A. K., Dutzan, N., Silva, N., Burleson, J. A., Strausbaugh, L. D., et al. (2013). The subgingival microbiome in health and periodontitis and its relationship with community biomass and inflammation. ISME J. 7, 1016-1025. doi: 10.1038/ismej.2012.174

Ahn, J., Yang, L., Paster, B. J., Ganly, I., Morris, L., Pei, Z., et al. (2011). Oral microbiome profiles: 16S rRNA pyrosequencing and microarray assay comparison. PLoS ONE 6:e22788. doi: 10.1371/journal.pone.0022788

Alcaraz, L. D., Belda-Ferre, P., Cabrera-Rubio, R., Romero, H., Simón-Soro, A., Pignatelli, M., et al. (2012). Identifying a healthy oral microbiome through metagenomics. Clin. Microbiol. Infect. 18(Suppl. 4), 54-57. doi: 10.1111/j.1469-0691.2012.03857.x

Andersson, A. F., Lindberg, M., Jakobsson, H., Bäckhed, F., Nyrén, P., and Engstrand, L. (2008). Comparative analysis of human gut microbiota by barcoded pyrosequencing. PLoS ONE 3:e2836. doi: 10.1371/journal.pone.0002836

Angelov, N., Aprecio, R. M., Kettering, J., Lundgren, T., Riggs, M., and Egelberg, J. (2009). Recovery of putative pathogens from paper point sampling at different depths of periodontal lesions. Clin. Cosmet. Investig. Dent. 1, 1-5. doi: 10.2147/CCIDE.S4737

Benítez-Páez, A., Belda-Ferre, P., Simón-Soro, A., and Mira, A. (2014). Microbiota diversity and gene expression dynamics in human oral biofilms. BMC Genomics. 15:311. doi: 10.1186/1471-2164-15-311

Caporaso, J. G., Bittinger, K., Bushman, F. D., DeSantis, T. Z., Andersen, G. L., and Knight, R. (2010). PyNAST: a flexible tool for aligning sequences to a template alignment. Bioinformatics 26, 266-267. doi: 10.1093/bioinformatics/btp636

Chakravorty, S., Helb, D., Burday, M., Connell, N., and Alland D. A. (2007). Detailed analysis of $16 \mathrm{~S}$ ribosomal RNA gene segments for the diagnosis of pathogenic bacteria. J. Microbiol. Methods 69, 330-339. doi: 10.1016/j.mimet.2007.02.005

Chao, A. (1984). Non-parametric estimation of the number of classes in a population. Scand. J. Statist. 11, 265-270.

Chen, L., Qin, B., Du, M., Zhong, H., Xu, Q., Li, Y., et al. (2015). Extensive description and comparison of human supra-gingival microbiome in root caries and health. PLOS ONE 10:e0117064. doi: 10.1371/journal.pone. 0117064

Cortelli, J. R., Fernandes, C. B., Costa, F. O., Cortelli, S. C., Kajiya, M., Howell, S. C. et al. (2012). Detection of periodontal pathogens in newborns and children with mixed dentition. Eur. J. Clin. Microbiol. Infect. Dis. 31, 1041-1050. doi: 10.1007/s10096-011-1405-9

Crielaard, W., Zaura, E., Schuller, A. A., Huse, S. M., Montijn, R. C., and Keijser, B. J. (2011). Exploring the oral microbiota of children at various developmental stages of their dentition in the relation to their oral health. BMC Med. Genomics. 4:22. doi: 10.1186/1755-8794-4-22

Diaz, P. I. (2012). Microbial diversity and interactions in subgingival biofilm communities. Front. Oral Biol. 15, 17-40. doi: 10.1159/000329669

Edgar, R. C. (2010). Search and clustering orders of magnitude faster than BLAST. Bioinformatics 26, 2460-2461. doi: 10.1093/bioinformatics/btq461
Supplementary Figure S1 | Ten rarefaction curves generated from subgingival microbiome profiles of five healthy children based on two performances of paper point sampling before (Mode A) and after (Mode B) supragingival cleansing with a sterile cotton pellet (colors designate sampling modes).

Supplementary Table S1 | Barcoded primer sequences used in this study: PCR ampicons were sequenced from the Titanium A adaptor (CCATCTCATCCCTGCGTGTCTCCGAC), followed by a 4 bases key sequence (TCAG) and the barcode. The reverse primer was used with the Titanium B adaptor (CCTATCCCCTGTGTGCCTTGGCAGTC), the key sequence and the target specific sequence but without barcode sequence.

Fierer, N., Lauber, C. L., Zhou, N., McDonald, D., Costello, E. K., and Knight, R. (2010). Forensic identification using skin bacterial communities. Proc. Natl. Acad. Sci. U.S.A. 107, 6477-6481. doi: 10.1073/pnas.10001 62107

Geibel, M. A. (2006). Development of a new micro-endoscope for odontological application. Eur. J. Med. Res. 11, 123-127.

Gohler, A., Hetzer, A., Holtfreter, B., Geisel, M. H., Schmidt, C. O., Steinmetz, I., et al. (2014). Quantitative molecular detection of putative periodontal pathogens in clinically healthy and periodontally diseased subjects. PLOS ONE 9:e99244. doi: 10.1371/journal.pone.0099244

Gomar-Vercher, S., Cabrera-Rubio, R., Mira, A., Montiel-Company, J. M., and Almerich-Silla, J. M. (2014). Relationship of children's salivary microbiota with their caries status: a pyrosequencing study. Clin. Oral Investig. 18, 2087-2094. doi: 10.1007/s00784-014-1200-y

Griffen, A. L., Beall, C. J., Campbell, J. H., Firestone, N. D., Kumar, P. S., Yang, Z. K., et al. (2012) Distinct and complex bacterial profiles in human periodontitis and health revealed by $16 \mathrm{~S}$ pyrosequencing. ISME J. 6, 1176-1185.

Griffen, A. L., Beall, C. J., Firestone, N. D., Gross, E. L., Difranco, J. M., Hardman, J. H., et al. (2011). CORE: a phylogenetically-curated 16S rDNA database of the core oral microbiome. PLoS ONE 6:e19051. doi: 10.1371/journal.pone.0019051

Hartroth, B., Seyfahrt, I., and Conrads, G. (1999). Sampling of periodontal pathogens by paper points: evaluation of basic parameters. Oral Microbiol. Immunol. 14, 326-330. doi: 10.1034/j.1399-302X.1999.140510.x

Huse, S. M., Dethlefsen, L., Huber, J. A., Mark Welch, D., Relman, D. A., and Sogin, M. L. (2008). Exploring microbial diversity and taxonomy using SSU rRNA hypervariable tag sequencing. PLoS Genet. 4:e1000255. doi: 10.1371/journal.pgen.1000255

Jenkinson, H. F. (2011). Beyond the oral microbiome. Environ. Microbiol. 13, 3077-3087. doi: 10.1111/j.1462-2920.2011.02573.x

Jervøe-Storm, P. M., Alahdab, H., Koltzscher, M., Fimmers, R., and Jepsen, S. (2007). Comparison of curet and paper point sampling of subgingival bacteria as analyzed by real-time polymerase chain reaction. J. Periodontol. 78, 909-917. doi: 10.1902/jop.2007.060218

Jünemann, S., Prior, K., Szczepanowski, R., Harks, I., Ehmke, B., Goesmann, A., et al. (2012). Bacterial community shift in treated periodontitis patients revealed by ion torrent $16 \mathrm{~S}$ rRNA gene amplicon sequencing. PLoS ONE 7:e41606. doi: 10.1371/journal.pone.0041606

Keijser, B. J., Zaura, E., Huse, S. M., van der Vossen, J. M., Schuren, F. H., Montijn, R. C., et al. (2008). Pyrosequencing analysis of the oral microflora of healthy adults. J. Dent. Res. 87, 1016-1020. doi: 10.1177/154405910808701104

Lazarevic, V., Whiteson, K., Hernandez, D., François, P., and Schrenzel, J. (2010). Study of inter- and intra-individual variations in the salivary microbiota. BMC Genomics. 11:523. doi: 10.1186/1471-2164-11-523

Ledder, R. G., Gilbert, P., Huws, S. A., Aarons, L., Ashley, M. P., Hull, P. S., et al. (2007). Molecular analysis of the subgingival microbiota in health and disease. Appl. Environ. Microbiol. 73, 516-523. doi: 10.1128/AEM.01419-06

Li, K., Bihan, M., and Methé, B. A. (2013). Analyses of the stability and core taxonomic memberships of the human microbiome. PLoS ONE 8:e63139. doi: 10.1371/journal.pone.0063139

Li, K., Bihan, M., Yooseph, S., and Methé, B. A. (2012). Analyses of the microbial diversity across the human microbiome. PLoS ONE 7:e32118. doi: 10.1371 /journal.pone.0032118 
Lif Holgerson, P., Ohman, C., Ronnlund, A., and Johansson, I. (2015). Maturation of Oral Microbiota in Children with or without Dental Caries. PLoS ONE 10:e0128534. doi: 10.1371/journal.pone.0128534

Ling, Z., Kong, J., Jia, P., Wei, C., Wang, Y., Pan, Z., et al. (2010). Analysis of oral microbiota in children with dental caries by PCR-DGGE and barcoded pyrosequencing. Microb. Ecol. 60, 677-690. doi: 10.1007/s00248-010-9712-8

Ling, Z., Liu, X., Wang, Y., Li, L., and Xiang, C. (2013). Pyrosequencing analysis of the salivary microbiota of healthy Chinese children and adults. Microb. Ecol. 65, 487-495. doi: 10.1007/s00248-012-0123-x

Lozupone, C., Lladser, M. E., Knights, D., Stombaugh, J., and Knight, R. (2011). UniFrac: an effective distance metric for microbial community comparison. ISME J. 5, 169-172. doi: 10.1038/ismej.2010.133

Luo, A. H., Yang, D. Q., Xin, B. C., Paster, B. J., and Qin, J. (2012). Microbial profiles in saliva from children with and without caries in mixed dentition. Oral Dis. 18, 595-601. doi: 10.1111/j.1601-0825.2012.01915.x

Moissl, C., Rachel, R., Briegel, A., Engelhardt, H., and Huber, R. (2005). The unique structure of archaeal 'hami', highly complex cell appendages with nano-grappling hooks. Mol. Microbiol. 56, 361-370. doi: $10.1111 / j .1365-2958.2005 .04294 . x$

Moissl, C., Rudolph, C., and Huber, R. (2002). Natural communities of novel archaea and bacteria with a string-of-pearls-like morphology: molecular analysis of the bacterial partners. Appl. Environ. Microbiol. 68, 933-937. doi: 10.1128/AEM.68.2.933-937.2002

Moissl, C., Rudolph, C., Rachel, R., Koch, M., and Huber, R. (2003). In situ growth of the novel SM1 euryarchaeon from a string-of-pearls-like microbial community in its cold biotope, its physical separation and insights into its structure and physiology. Arch. Microbiol. 180, 211-217. doi: 10.1007/s00203-003-0580-1

Offenbacher, S., Barros, S. P., and Beck, J. D. (2008). Rethinking periodontal inflammation. J. Periodontol. 79(8 Suppl.), 1577-1584. doi: 10.1902/jop.2008.080220

Papaioannou, W., Gizani, S., Haffajee, A. D., Quirynen, M., Mamai-Homata, E., and Papagiannoulis, L. (2009). The microbiota on different oral surfaces in healthy children. Oral Microbiol. Immunol. 24, 183-189. doi: 10.1111/j.1399-302X.2008.00493.x

Park, O. J., Yi, H., Jeon, J. H., Kang, S. S., Koo, K. T., Kum, K. Y., et al. (2015). Pyrosequencing analysis of subgingival microbiota in distinct periodontal conditions. J. Dent. Res. 94, 921-927. doi: 10.1177/0022034515583531

Paster, B. J., Boches, S. K., Galvin, J. L., Ericson, R. E., Lau, C. N., Levanos, V. A., et al. (2001). Bacterial diversity in human subgingival plaque. J. Bacteriol. 183, 3770-3783. doi: 10.1128/JB.183.12.3770-3783.2001

Pihlstrom, B. L., Michalowicz, B. S., and Johnson, N. W. (2005). Periodontal diseases. Lancet 366, 1809-1820. doi: 10.1016/S0140-6736(05)67728-8

Price, L. B., Liu, C. M., Melendez, J. H., Frankel, Y. M., Engelthaler, D., Aziz, M., et al. (2009). Community analysis of chronic wound bacteria using $16 \mathrm{~S}$ rRNA gene-based pyrosequencing: impact of diabetes and antibiotics on chronic wound microbiota. PLoS ONE 4:e6462. doi: 10.1371/journal.pone.0006462

R Core Team, R. (2015). A Language and Environment for Statistical Computing. Vienna: R Foundation for Statistical Computing.
Sahl, E. F., Henkin, J. M., and Angelov, N. (2014). Recovery of putative periodontal pathogens from curette sampling at different depths of periodontal lesions: an in vivo cross-sectional clinical study. J. Int. Acad. Periodontol. 16, 78-85.

Shannon, C. (1948). A mathematical theory of communication. Bell Syst Tech, J. 27, 379-423, 623-656. doi: 10.1002/j.1538-7305.1948.tb00917.x

Simón-Soro, A., Tomás, I., Cabrera-Rubio, R., Catalan, M. D., Nyvad, B., and Mira, A. (2013). Microbial geography of the oral cavity. J. Dent. Res. 92, 616-621. doi: $10.1177 / 0022034513488119$

Simpson, E. H. (1949). Measurement of diversity. Nature 163:688

Siqueira, J. F. Jr., Fouad, A. F., and Rocas, I. N. (2012). Pyrosequencing as a tool for better understanding of human microbiomes. J. Oral Microbiol. 4, 10743-10758. doi: 10.3402/jom.v4i0.10743

Socransky, S. S., and Haffajee, A. D. (2005). Periodontal microbial ecology. Periodontology 2000 38, 135-187. doi: 10.1111/j.1600-0757.2005.00107.x

Stahringer, S. S., Clemente, J. C., Corley, R. P., Hewitt, J., Knights, D., Walters, W. A., et al. (2012). Nurture trumps nature in a longitudinal survey of salivary bacterial communities in twins from early adolescence to early adulthood. Genome Res. 22, 2146-2152. doi: 10.1101/gr.140608.112

Teles, F. R., Haffajee, A. D., and Socransky, S. S. (2008). The reproducibility of curet sampling of subgingival biofilms. J. Periodontol. 79, 705-713. doi: 10.1902/jop.2008.070424

Trajanoski, S., Klug, B., Klymiuk, I., Bozic, M., Grube, M., Wimmer, G., et al. (2013). Next-generation sequencing in microbiome analysis: factors affecting reproducibility of repeated biofilm sampling of the gingival sulcus of children. J. Dent. Oral Craniof. Epidemiol. 1, 34-46.

Wade, W. G. (2013). The oral microbiome in health and disease. Pharmacol. Res. 69, 137-143. doi: 10.1016/j.phrs.2012.11.006

Wang, Q., Garrity, G. M., Tiedje, J. M., and Cole, J. R. (2007). Naive Bayesian classifier for rapid assignment of rRNA sequences into the new bacterial taxonomy. Appl. Environ. Microbiol. 73, 5261-5267. doi: 10.1128/AEM.00062-07

Wu, G. D., Lewis, J. D., Hoffmann, C., Chen, Y. Y., Knight, R., Bittinger, K., et al. (2010). Sampling and pyrosequencing methods for characterizing bacterial communities in the human gut using $16 \mathrm{~S}$ sequence tags. BMC Microbiol. 10:206. doi: 10.1186/1471-2180-10-206

Xin, B. C., Luo, A. H., Qin, J., Paster, B. J., Xu, Y. L., Li, Y. L., et al. (2013). Microbial diversity in the oral cavity of healthy Chinese Han children. Oral Dis. 19, 401-405. doi: 10.1111/odi.12018

Conflict of Interest Statement: The authors declare that the research was conducted in the absence of any commercial or financial relationships that could be construed as a potential conflict of interest.

Copyright $\odot 2017$ Santigli, Trajanoski, Eberhard and Klug. This is an open-access article distributed under the terms of the Creative Commons Attribution License (CC $B Y)$. The use, distribution or reproduction in other forums is permitted, provided the original author(s) or licensor are credited and that the original publication in this journal is cited, in accordance with accepted academic practice. No use, distribution or reproduction is permitted which does not comply with these terms. 